\title{
Soft Physics in STAR
}

\author{
G. Van Buren ${ }^{\mathrm{a}}$ for the STAR Collaboration*

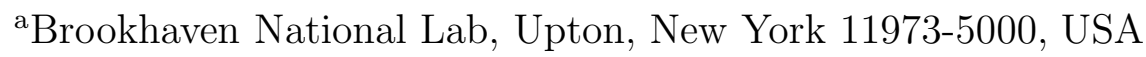

The STAR Experiment at RHIC is well-suited to making measurements of particle yields from relativistic nuclear collisions in the low transverse momentum, or "soft", regime. We present preliminary results on measurements of $\pi^{0}, \pi^{-}, \pi^{+}, K^{-}, K^{+}, K_{s}^{0}, \rho, K^{* 0}+\overline{K^{* 0}}, \mathrm{f}_{0}$, $p, \bar{p}, \phi, \Lambda, \bar{\Lambda}, \Xi^{-}, \bar{\Xi}^{+}, \Omega^{-}$, and $\bar{\Omega}^{+}$from the first two years of physics running at RHIC. An abundance of physics topics can be addressed by these measurements, some of which are discussed here along with the results.

\section{Introduction}

"The principles of thermodynamics are devised for giving a phenomenological account of the gross behavior of macroscopic physical systems, ... [interpreting] the average behavior of systems of many degrees of freedom." The ongoing search for a system of nuclear matter which can be described by a thermodynamic quark gluon plasma phase (QGP) must therefore involve a study of nuclear matter in bulk. For this reason, heavy ions with significant amounts of nuclear matter are used in laboratory experiments to find and study QGP. This also implies that QGP formation affects some bulk portion of the outgoing matter, most of which is in the "soft", low- $p_{T}$ region of phase space.

The STAR Experiment at RHIC has measured a rich variety of particle species and portions of their soft spectra in high energy $A u+A u$ and $p+p$ collisions [ 1, 2, 3, 4, 5, 6, 7, 8]. Because production, annihilation, and rescattering processes differ among the particle species, their abundances and spectra reflect on different aspects of the collision evolution. We present here a discussion of these aspects as elucidated by our measurements. We will follow an outline of the physics topics along the evolution from initial collisions to final reinteractions of the products.

\section{Inclusive Multiplicity and Mean Transverse Momenta}

Improved statistics in the second year of physics running at RHIC have given STAR the ability to measure unidentified charged hadron transverse momentum $\left(p_{T}\right)$ spectra from $0.2 \mathrm{GeV} / c$ to $\sim 12 \mathrm{GeV} / c$. These spectra are shown in Fig. 1 as a function of centrality for $A u+A u$ collisions. They have been corrected for decays and backgrounds through detailed simulation [ 9]. The corrections are $p_{T}$ and multiplicity dependent, but nowhere exceed $20 \%$. From these inclusive spectra we can measure total yields as well as $\left\langle p_{T}\right\rangle$. This is done

*For the full author list and acknowledgements, see Appendix "Collaborations" of this volume.

${ }^{\dagger} \mathrm{R}$. Tolman, The Principles of Statistical Mechanics, Oxford University Press (1938). 
by fitting the spectra with a power law of the form $C\left(1+p_{T} / p_{0}\right)^{-n}[10]$. The $p_{T^{-}}$integrated multiplicity requires extrapolation of the power law at the low- $p_{T}$ end, which comprises up to $21 \%$ of the yields. For the $5 \%$ most central events, our preliminary results indicate that the average charged hadronic multiplicity $\left\langle d N_{c h} / d \eta\right\rangle$ increases from $563 \pm 39$ to $687 \pm 69$ between $\sqrt{s_{\mathrm{NN}}}$ $=130$ and $200 \mathrm{GeV} \cdot\left\langle p_{T}\right\rangle$ is then obtained from the same power law, giving $0.520 \pm 2.3 \% \mathrm{GeV} / c$ at $\sqrt{s_{\mathrm{NN}}}=130 \mathrm{GeV}$ and $0.524 \pm 2.3 \% \mathrm{GeV} / c$ at $200 \mathrm{GeV}$. For both $\left\langle d N_{c h} / d \eta\right\rangle$ and $\left\langle p_{T}\right\rangle$ measurements the errors are dominated by systematics mostly common between the two energies and do not reflect possible relative variations between each other.

To put these measurements in context, a parameterization of the Color Glass Condensate (CGC) model [11] is shown fit to central $A+A\left\langle p_{T}\right\rangle$ at $\sqrt{s_{\mathrm{NN}}}=17$ and $130 \mathrm{GeV}$ in Fig. 2 [ 12]. While the CGC

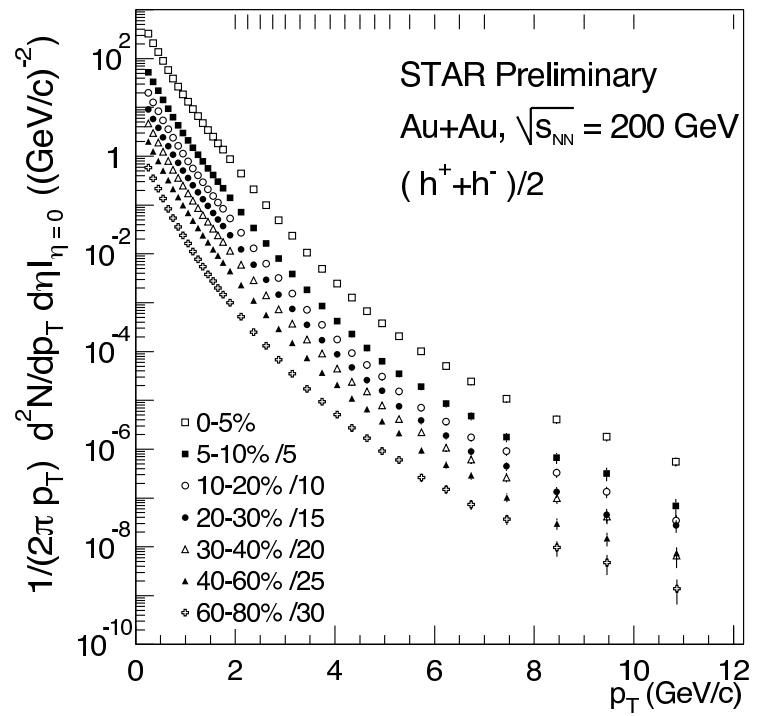

Figure 1: Transverse momentum spectra of hadrons from $\sqrt{s_{\mathrm{NN}}}=200 \mathrm{GeV} A u+A u$ over several centralities. Only statistical errors are shown. See Ref. [1] for systematic errors and more details. parameterization can fit these two data points, the $200 \mathrm{GeV}$ central $A u+A u\left\langle p_{T}\right\rangle$ datum falls well below the fit from the lower energies, despite anticipation that the model is most applicable in high energy central $A+A$ collisions. The CGC model has had numerous other successes [13], but may require supplemental considerations to congrue with these data [14].

Also evident in Fig. 2 are the differences in collision energy systematics for $\left\langle p_{T}\right\rangle$ between $A+A, e^{+}+e^{-}$and $p(\bar{p})+p$ data. This implies that $A+A$ is not just a simple superposition of nucleon+nucelon collisions [ 14], and argues against a universality in particle production [15] suggested from total particle yields [16].

\section{Baryon Number}

Conserved quantities must survive any evolution. The conserved net-baryon number is established from the incoming nuclei, but becomes spread over the rapidity interval by initial interactions (often called stopping, though not strictly stopped), and then additionally smeared by the rescattering processes; together these make up the

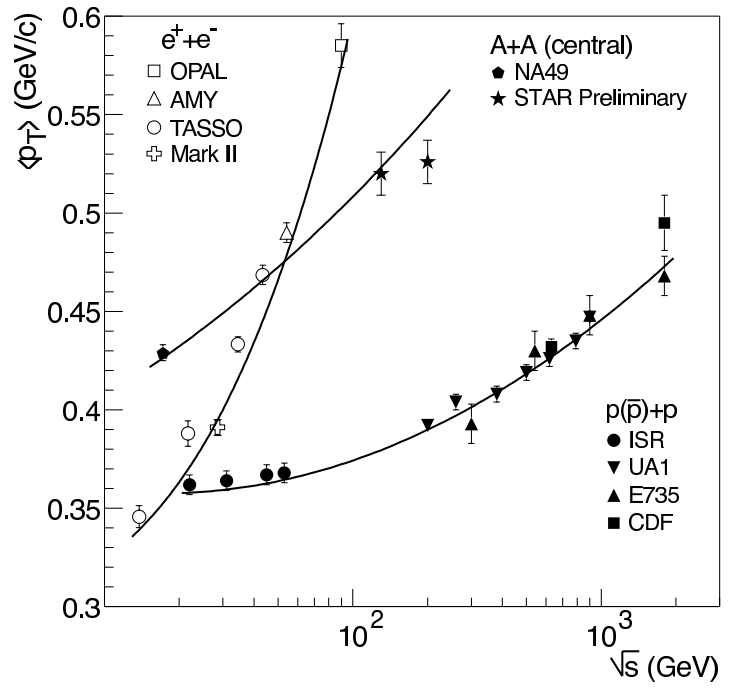

Figure 2: Mean transverse momenta of hadrons for central heavy ion, $e^{+}+e^{-}$, and $p(\bar{p})+p$ collisions versus energy [12]. Curves are CGC model for $A+A$, phenomenological parameterization for $p(\bar{p})+p$, and JETSET calculations (versus thrust axis) for $e^{+}+e^{-}$. STAR data errors are systematics-dominated. 
transport of baryon number from the nuclei to their final rapidities. The midrapidity baryon and antibaryon yields thus have folded into them these transport distributions along with components from baryon-antibaryon pair production (and annihilation, which may also contribute to transport) processes.

A characterization of the baryon-antibaryon differences is made in Fig. 3. This plot shows the antibaryon to baryon ratios at midrapidity for various species in central heavy ion collisions. It is clear from the plot that the ratios tend towards one with increasing strangeness content, and with increasing energy. The ratios are not corrected for feeddown, and the $p$ and $\bar{p}$ are essentially inclusive. These ratios indicate that the yield of baryons resulting from pair production processes has begun to dominate over those from transport at RHIC energies: the ratio of those contributions from inclusive (anti)protons in central $A u+A u$ is $\sim 2.5$ at $\sqrt{s_{\mathrm{NN}}}=130 \mathrm{GeV}$, derived from inclusive $\bar{p} / p=0.72 \pm 0.05$ (syst.), and $\sim 3.5$ at $200 \mathrm{GeV}$, from $\bar{p} / p=0.78 \pm 0.05$ (syst.). Inclusion of strange quarks in the baryons ap-

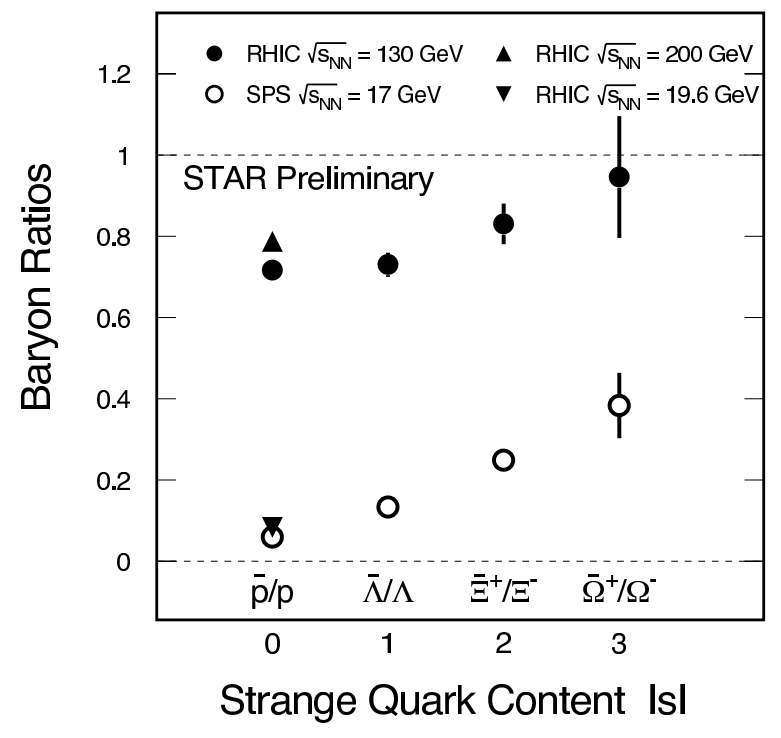

Figure 3: Midrapidity antibaryon to baryon ratios are shown for various species and at several energies of central heavy ion collisions. Only statistical errors are shown. Systematic errors range from $\sim 7 \%$ for $\bar{p} / p$ to $\sim 15 \%$ for $\bar{\Omega}^{+} / \Omega^{-}$in RHIC data.

pears to dilute the contributions of transport, enough to make the $\bar{\Omega}^{+} / \Omega^{-}$ratio consistent with 1 at RHIC; this is not the case at SPS energies where the contributions from transport are still evident in the $\Omega$ yields despite an initial net strange quark number of zero.

STAR has also measured most of these antibaryon to baryon ratios as a function of centrality and sees only a slight rise in the antiproton to proton ratio for peripheral collisions, and no significant changes for the other ratios [ 2, 3, 17]. This implies little change in the relevance of pair production processes for baryon and antibaryon production despite the changing collision geometry and participants.

A definitive measure of the baryon number transported to midrapidity comes from the difference between yields of baryons and antibaryons at midrapidity. This is represented by the net inclusive proton number shown in Fig. 4 (left). This number is clearly decreasing with increasing collision energy for large heavy ion collisions, while the yield of produced particles at midrapidity (represented in Fig. 4 (left) by the negative hadron yield) is monotonically increasing. This demonstrates the decreasing significance of baryon transport contributions to midrapidity yields and is not unexpected as the rapidity interval from the original nuclei is stretched. It is interesting that the baryon number transport is in fact still nonzero despite the interval of $\sim 5$ units in rapidity at $130 \mathrm{GeV}$ (also true for $\sim 6$ rapidity units at $200 \mathrm{GeV}$ ). Some understanding of this may come from the asymmetric $q$ versus $\bar{q}$ distributions even at low momentum fraction $x=p_{q, \bar{q}} / p_{N}$ within the incoming nucleons [ 18]. Several model predictions are also shown for the centrality plot, with HIJING matching peripheral data best, and HIJING/B $\bar{B}$ (which is intended to enhance 

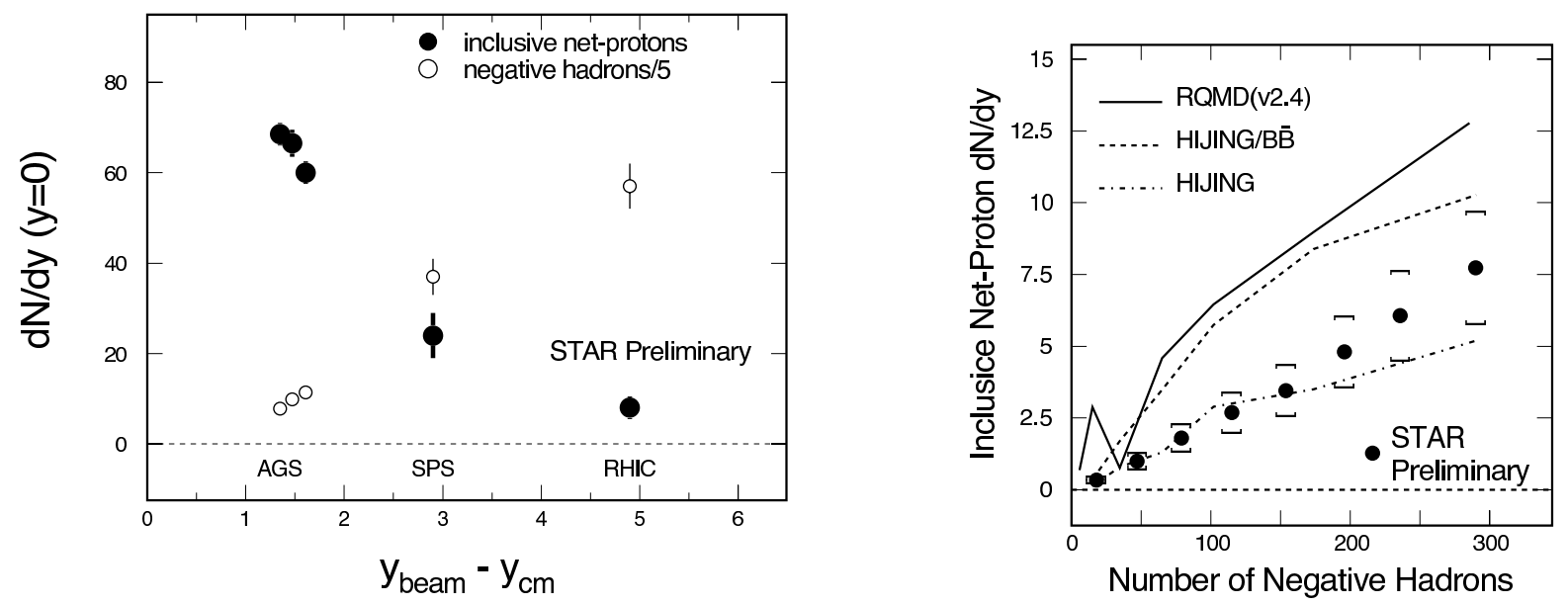

Figure 4. The net protons at midrapidity are shown versus collision energy for central collisions along with the negative hadrons (left, statistical errors only), and versus centrality for $\sqrt{s_{\mathrm{NN}}}=130 \mathrm{GeV}$ along with model predictions (right, errors systematics-dominated).

the stopping [ 19]) more closely approaching the central data. RQMD overpredicts the transport contributions.

\section{Particle Production}

Long-standing arguments exist for extraordinary hadron yields should a QGP form, such as strangeness phase space saturation (enhanced yields over a solely hadronic evolution) [ 20] and $\mathrm{J} / \psi$ suppression [ 21]. While studies of hadron production in the low $p_{T}$ region of phase space where a QGP is likely to manifest are appropriate for these searches, it should be understood that experiments often measure yields after any QGP has condensed, at the end of all hadrochemical reactions which can potentially hide earlier irregularities (equilibration of a hadron gas, for example).

It is all the more important that we study the particle production to learn what we can about the system and its evolution. STAR is well-suited for this task, capable of identifying and measuring spectra and yields for a plethora of particle species. Results on the following species have been presented at this conference: $\pi^{0}, \pi^{-}, \pi^{+}, K^{-}, K^{+}, K_{s}^{0}$, $\rho, K^{* 0}+\overline{K^{* 0}}, \mathrm{f}_{0}, p, \bar{p}, \phi, \Lambda, \bar{\Lambda}, \Xi^{-}, \bar{\Xi}^{+}, \Omega^{-}$, and $\bar{\Omega}^{+}$[ [2, 3, 4, 5, 6, 7, 8]. The impressive preliminary spectra and fits for several of these species from the $\sqrt{s_{\mathrm{NN}}}=200 \mathrm{GeV}$ data can be seen in Fig. 5, and in Table 1 where the $m_{T}$ reach of the measurements and results of the fits are presented. Our observations of the $K^{* 0}, \rho$, and $\mathrm{f}_{0}$ are the first in heavy ion collisions.

One can attempt to obtain a global characterization of particle production by comparing the yields to a model which describes the bulk properties of the system through a limited set of parameters. A thermal, chemical equilibrium model is one such statistical model, and we can examine how this model describes our $\sqrt{s_{\mathrm{NN}}}=130 \mathrm{GeV}$ midrapidity data, mindful of any caveats [ 22]. In the particular model we choose, free parameters include the temperature $(T)$, light quark chemical potential $\left(\mu_{q}\right)$, strange quark chemical potential $\left(\mu_{s}\right)$, and strangeness saturation factor $\left(\gamma_{s}\right.$, where values less than one indicate incomplete saturation) [ 23]. Shown in Fig. 6 is a fit excluding the $K^{*}$, $\phi, \Xi$, and $\Omega$ results $\left(\chi^{2} /\right.$ dof $\left.=1.53\right)$. From this we can compare the excluded data 

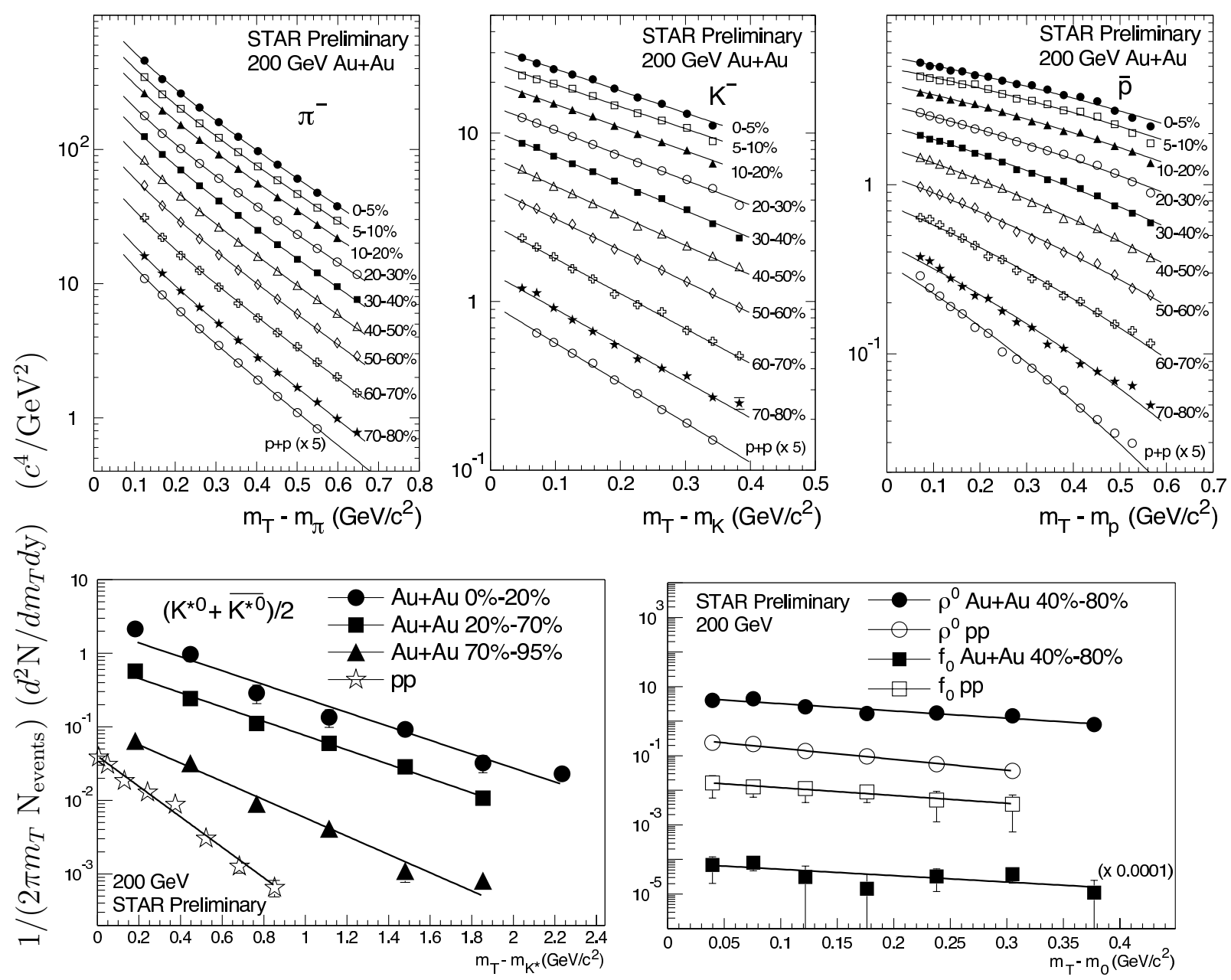

$\frac{\ddot{0}}{0}$
$\frac{0}{0}$
$\bar{z}$
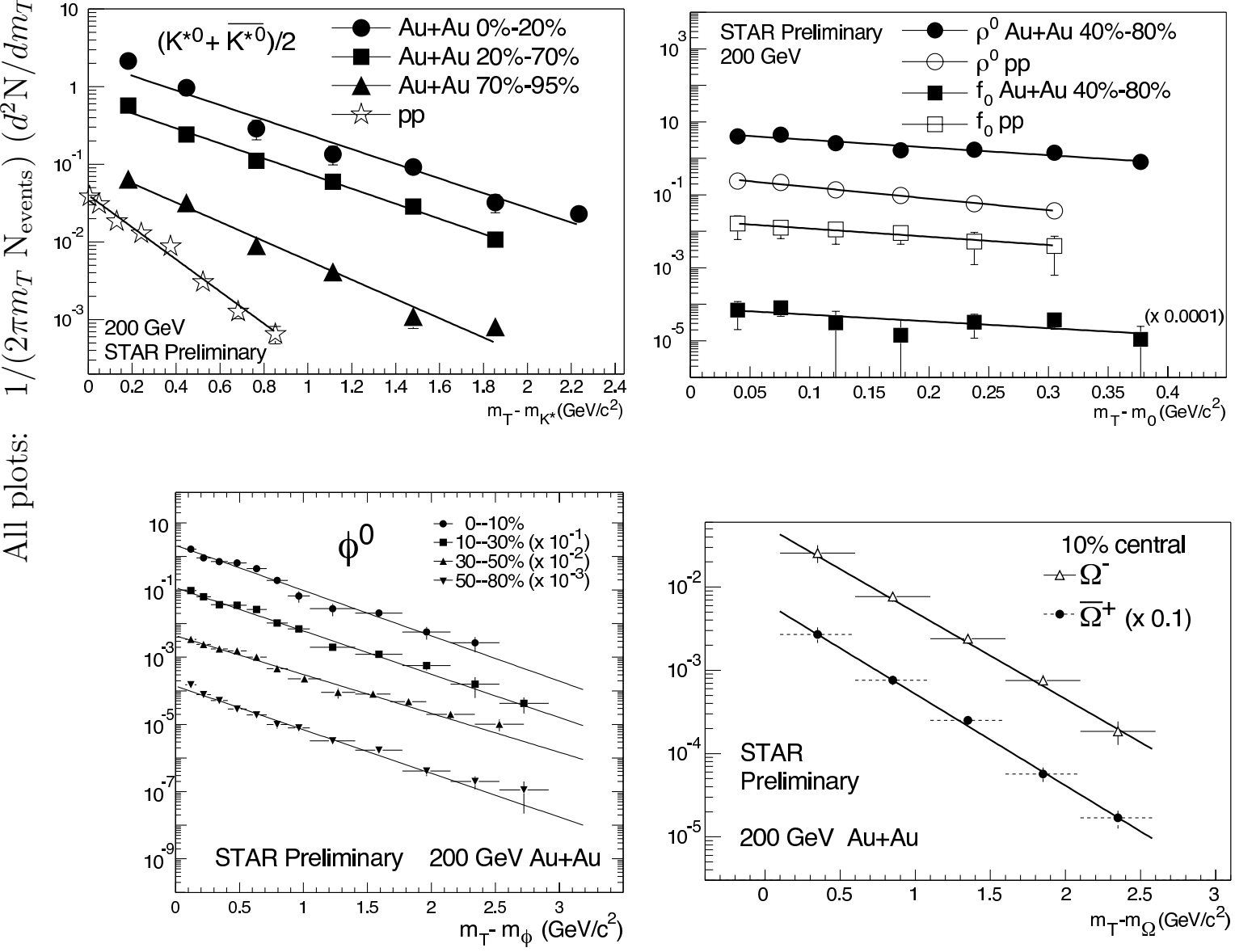

Figure 5. A variety of preliminary midrapidity spectra from $\sqrt{s_{\mathrm{NN}}}=200 \mathrm{GeV} A u+A u$ and $p+p$ collisions in STAR are plotted, including those of $\pi^{-}, K^{-}$, and inclusive $\bar{p}$ [2] $,\left(K^{* 0}+\overline{K^{* 0}}\right) / 2, \rho$, and $\mathrm{f}_{0}$ [ [6], $\phi$ [ [0], and $\Omega$ [ $[$. Only statistical errors are shown. See respective references for systematic errors and further details. All fits are $m_{T}$ exponentials except for Bose-Einstein on $\pi^{-}$and $p_{T}$ Gaussian on $\bar{p}$; the fit results can be found in Table 1 for selected centralities. 
to the outcome of the fit based on the included species, demonstrating how well the model matches all of the data. Including all ratios results in $\chi^{2} /$ dof $=1.68$. In either fit, the preferred value of $\gamma_{s}$ is close to 1 . Other chemical equilibrium models have also predicted and fit most of these particle yields rather well [24], converging on similar values for the thermodynamic parameters (with a temperature range from $165 \pm 7$ $\mathrm{MeV}$ to $179 \pm 9 \mathrm{MeV}$, for example). While these models may or may not be applicable, and good fits are neither new (even seen in $e^{+}+e^{-}$and $p(\bar{p})+p$ data, albeit without strangeness saturation) nor absolute proof of equilibrium [25], our results are provocative: 1) the fits indicate approximate strangeness phase space saturation for central collisions, and 2) recent lattice QCD calculations for non-zero chemical potentials have determined a similar temperature of $172 \pm 3 \mathrm{MeV}$ in this region of the phase boundary between hadronic matter and QGP [ 26.

Table [1 shows the preliminary results of spectra measurements in STAR from $A u+A u$ at $\sqrt{s_{\mathrm{NN}}}$ $=200 \mathrm{GeV}$ for $\pi^{-}, K^{-}, \rho$, $K^{* 0}+\overline{K^{* 0}}, \bar{p}, \mathrm{f}_{0}, \phi, \Omega^{-}$, and $\bar{\Omega}^{+}[$ 2, $4,5,6$. A preliminary $p_{T^{-}}$ independent muon contamination correction has been applied to the pions. More data is necessary to

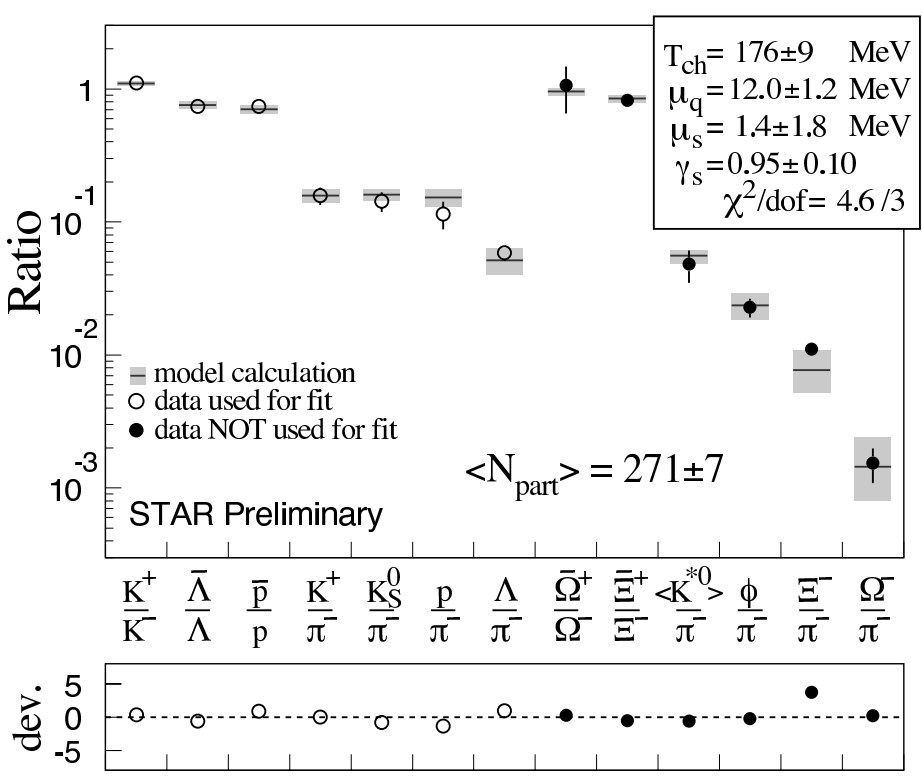

Figure 6: A fit to the STAR midrapidity $15 \%$ central $130 \mathrm{GeV}$ $A u+A u$ data with a thermal statistical model [23] is shown.

tightly constrain a chemical fit.

\section{Transverse Momentum Systematics}

Transverse radial flow can be gauged through the mass dependence of either inverse slope parameters $(T)$ of spectra fits (inversely proportional to the steepness of yield fall-off in $m_{T}$ ) [28], or $\left\langle p_{T}\right\rangle$ [ 29] of identified spectra. These are shown for various STAR data from central $A u+A u$ collisions at $130 \mathrm{GeV}$ in Figs. 7 and 8(left). It is important that the inverse slope is measured over a large range of the produced spectrum to permit valid comparisons, and this holds for the data shown in Fig. 7. Here it can be seen that the rising trend versus mass expected from transverse radial flow [28] is seen for the lighter species,

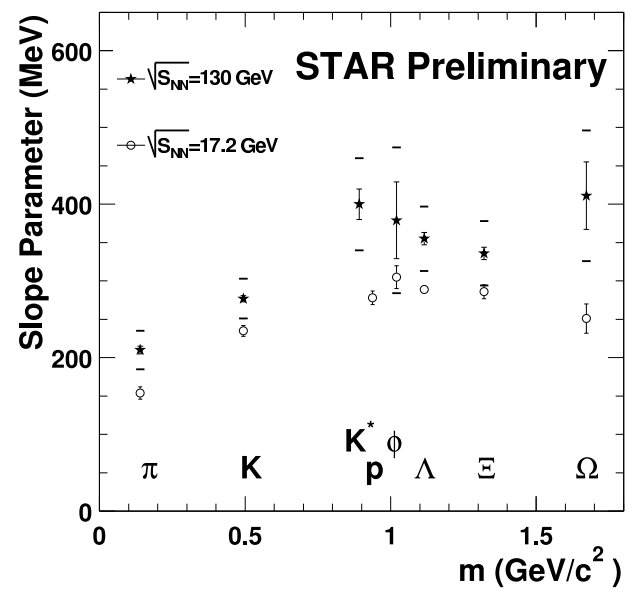

Figure 7: Inverse slope parameters as a function of particle mass for SPS [27, 28] and RHIC are shown with statistical error bars, and systematic error brackets for RHIC data only. 

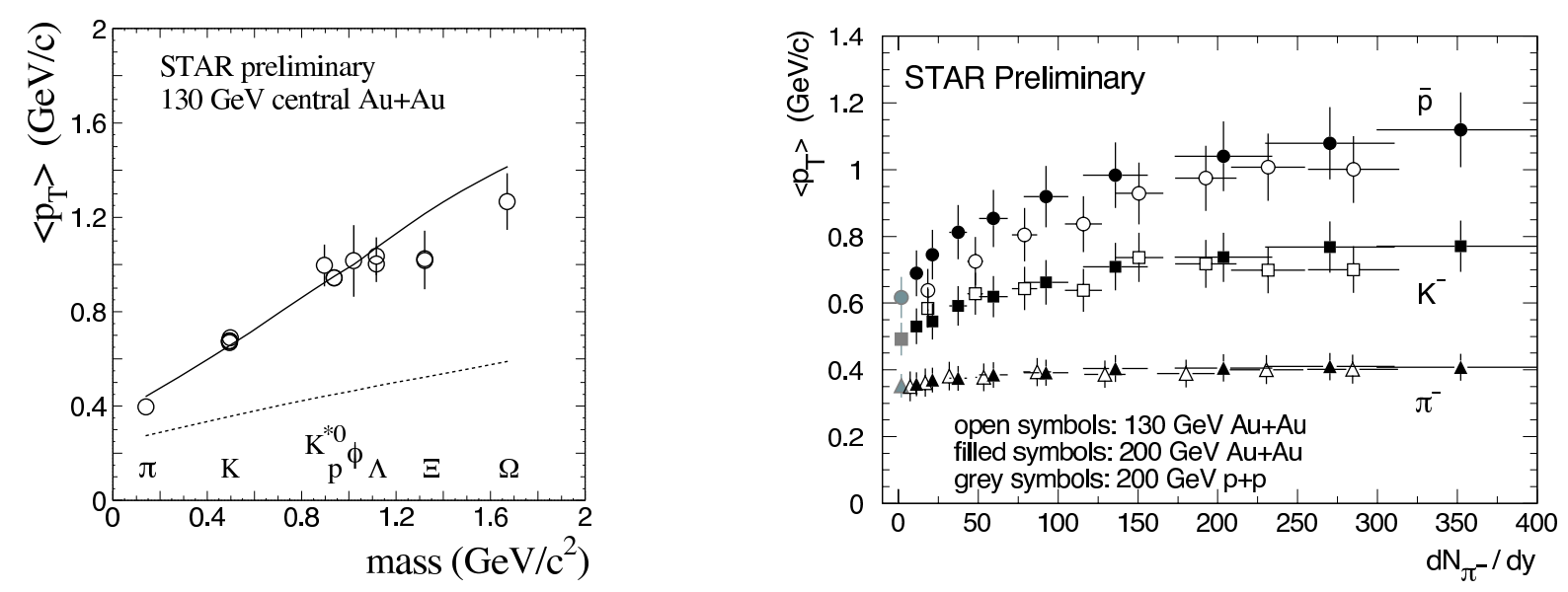

Figure 8. Mean transverse momenta of identified species versus mass (left) for $\sqrt{s_{\mathrm{NN}}}=130 \mathrm{GeV}$ $A u+A u$ collisions along with blast wave model fit (upper curve: $T_{\text {fo }} \simeq 100 \mathrm{MeV},\left\langle\beta_{T}\right\rangle \simeq 0.55$ ) and zero flow curve (lower curve: $T_{\mathrm{fo}} \simeq 100 \mathrm{MeV}, \beta_{T} \equiv 0$ ). $\left\langle p_{T}\right\rangle$ of $\pi^{-}, K^{-}$, and $\bar{p}$ are mapped against multiplicity (right) for $\sqrt{s_{\mathrm{NN}}}=130 \mathrm{GeV} A u+A u$, and $200 \mathrm{GeV} A u+A u$ and $p+p$. All errors are systematics-dominated.

but that the strange baryons do not clearly follow this trend. Further discussion of these systematics can be found in Ref. [12].

The same behavior is exhibited in Fig. \&(left) by the central $\left\langle p_{T}\right\rangle$ values. Fig. 8(right) shows how the $\left\langle p_{T}\right\rangle$ develops as a function of centrality for $\pi^{-}, K^{-}$, and $\bar{p}$ at RHIC. Going from $p+p$ data, through peripheral to central $A u+A u$ data shows a smooth rise consistent with the onset and buildup of transverse radial flow.

A blast wave [30] fit to the central $\pi^{-}, K^{-}$, and $\bar{p}$ spectra provides a transverse radial flow velocity of $\left\langle\beta_{T}\right\rangle \simeq 0.55$ at $130 \mathrm{GeV}$, and $\sim 0.60$ at $200 \mathrm{GeV}$. These flow velocities appear higher than the values of $\sim 0.50$ obtained at the SPS [31], indicating stronger flow. However, some deviation from the global blast wave fit is seen in the multistrange baryons at RHIC [ [⿴囗十). One additional note is that the fit kinetic freezeout temperature parameters show at most a mild decrease going from SPS $\left(T_{\mathrm{fo}} \simeq 110 \mathrm{MeV}\right)$ to $\mathrm{RHIC}\left(T_{\mathrm{fo}} \simeq 100 \mathrm{MeV}\right)$ energies.

\section{In-Medium Decay}

One exciting prospect is the possibility to probe the time scale of hadronic rescattering via in-medium decay. The principle here is that the daughters of a particle which decays during the rescattering process have the opportunity to rescatter. With altered momenta in either or both of the decay daughters, reconstruction of the parent particle through invariant mass of the daughters is no longer possible. Particles whose decay time scales are on the order of the lifetime of the rescattering period (perhaps a few $\mathrm{fm} / \mathrm{c}$ ) might then have a suppression in their reconstructed yield proportional to that lifetime. One must, however, make some assumption as to the expected yield in order to determine the fraction lost. There also remains an argument that some additional production of the parent resonances might occur during rescattering. This would dilute the measurement of the fraction lost.

To gain some further understanding, STAR has measured several short-lived species, and has more to come. As an example, Fig. 9 shows the $\pi^{+} \pi^{-}$invariant mass spectra 

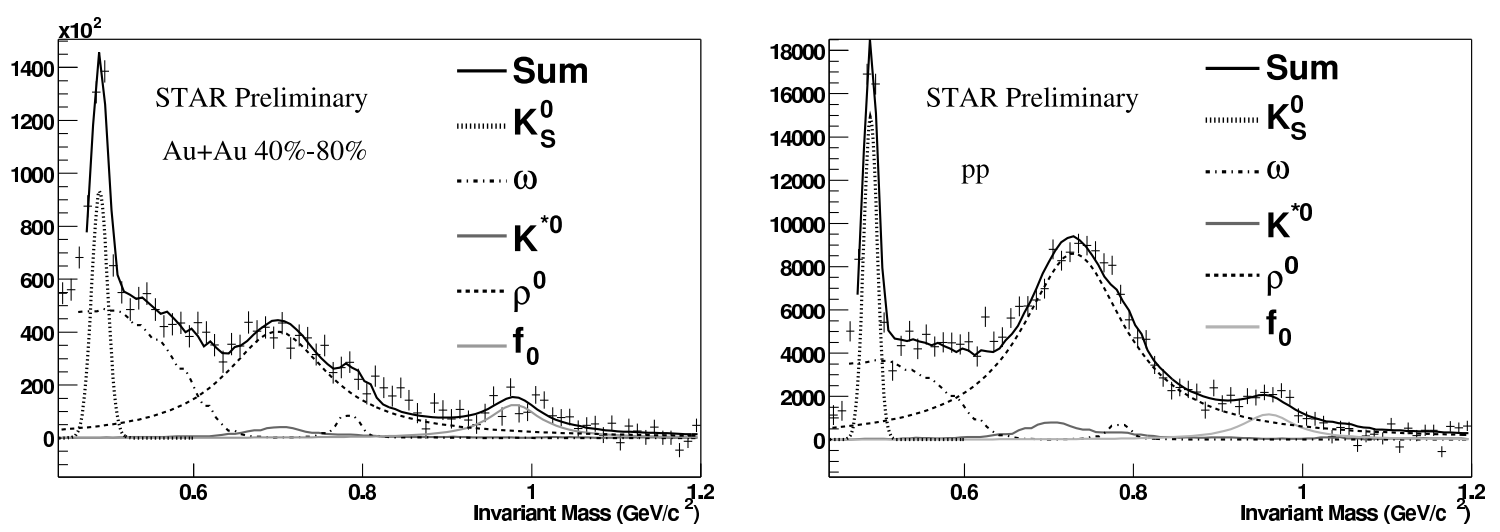

Figure 9. The $\pi^{+} \pi^{-}$invariant mass distributions after background subtraction for the $40 \%-80 \%$ of the hadronic $A u+A u$ cross section (left) and for $p+p$ interactions (right) at $\sqrt{s_{\mathrm{NN}}}=200 \mathrm{GeV}$.

from $A u+A u$ and $p+p$ data at $\sqrt{s_{\mathrm{NN}}}=200 \mathrm{GeV}$. Here we see evidence for such short-lived resonances as the $K^{* 0}(892), \rho(770)$, and $\mathrm{f}_{0}(980)$. Work is also in progress to measure the $\eta^{0}, K^{*+,-}, \Delta, \Lambda(1520)$, and $\Sigma(1385)$ (for the first time in heavy ion collisions), along with the $\phi$, which was presented at this conference [5].

In particular, the yield of the $K^{* 0}$ has been obtained [ 6] and was already shown in Fig. 6 to not differ notably from a thermal model for central $A u+A u$ collisions. A more detailed comparison can be made by taking the ratio of the $K^{* 0}$ yield to that of the $K$ and $\phi$. These ratios are shown in Fig. 10 along with the same ratios for other colliding systems and energies. On this level, it appears that $K^{* 0} / K$ is to some degree suppressed in heavy ion collisions at RHIC energies, while the $\phi / K^{* 0}$ ratio appears high. Together these hint at a slightly low value for the yield of the $K^{* 0}$. This could be construed as loss due to in-medium decay. However, with a $c \tau$ shorter than the size of a $A u$ nucleus (and average velocities considerably slower than $c$ ), such losses are actually rather small. This is consistent with either a very short lifetime of the rescattering period [ [32], or a loss rate only slightly higher than the regeneration rate during rescattering.

This is only one in a series of observables which may help us understand the time scales involved in the collision evolution. STAR is actively working on measurements via other techniques such as HBT correlations and balance functions to provide more handles on time and size scales. Several results from these studies have also been presented at this conference [33].

\section{Photonic $\pi^{0}$ Decay}

A notable demonstration of STAR's capabilities comes in measuring the spectra of $\pi^{0}$ through the $2 \gamma$ decay channel, via the subsequent conversion of each $\gamma$ into

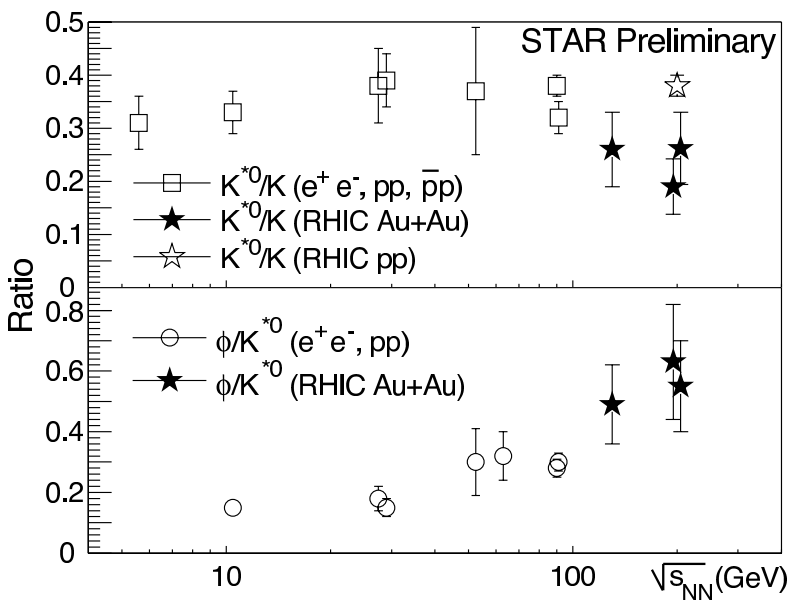

Figure 10: $K^{* 0} / K$ and $\phi / K^{* 0}$ yield ratios as a function of colliding system and energy. For more details, see Ref. [6].

an $e^{+} e^{-}$pair in the structural material of the detectors. Details of the analysis for this 4-body final state can be found in Ref. [ ]]. 
Spectra for the $\pi^{0}$ are measured for several centralities. As seen in Fig. 11, the shape compares well with the soft negative and positive hadron spectra for central $A u+A u$ collisions at $130 \mathrm{GeV}$ (which are dominated by $\pi^{-}$and $\pi^{+}$respectively at low $p_{T}$ ). An overall normalization uncertainty of $\pm 49 \%$ not indicated in the shown errors remains. It will be exciting to see what can be done with the improvements available for making this measurement in the $200 \mathrm{GeV}$ data and beyond from STAR. One addressable topic may be determination of the $\pi^{0} \rightarrow \gamma \gamma$ contributions to the photon spectra. This and the $\eta^{0} \rightarrow \gamma \gamma$ decay alone account for $>97 \%$ of the inclusive photon spectra in $\mathrm{Pb}+\mathrm{Pb}$ collisions at $\sqrt{s_{\mathrm{NN}}}=17 \mathrm{GeV}[34]$. STAR measures the inclusive $\gamma$ spectra (currently via the method outlined above using the TPC, and ahead with the electromagnetic calorimeter), and has observed an $\eta^{0}$ signal [ 7]; the future looks intriguing.

\section{Summary}

Data from the STAR Experiment in the soft regime at midrapidity probe bulk properties of the evolution of heavy ion collisions at RHIC energies. Net baryons from these collisions continue to decrease with increasing energy, but are still nonzero. This means that pair production processes behind baryon yields are now dominant, but do not account for all baryon number; there must remain some transport of baryon number even over $\sim 5$ and $\sim 6$ units of rapidity at 130 and $200 \mathrm{GeV}$ respectively. Also, the $\left\langle p_{T}\right\rangle$ systematics of inclusive

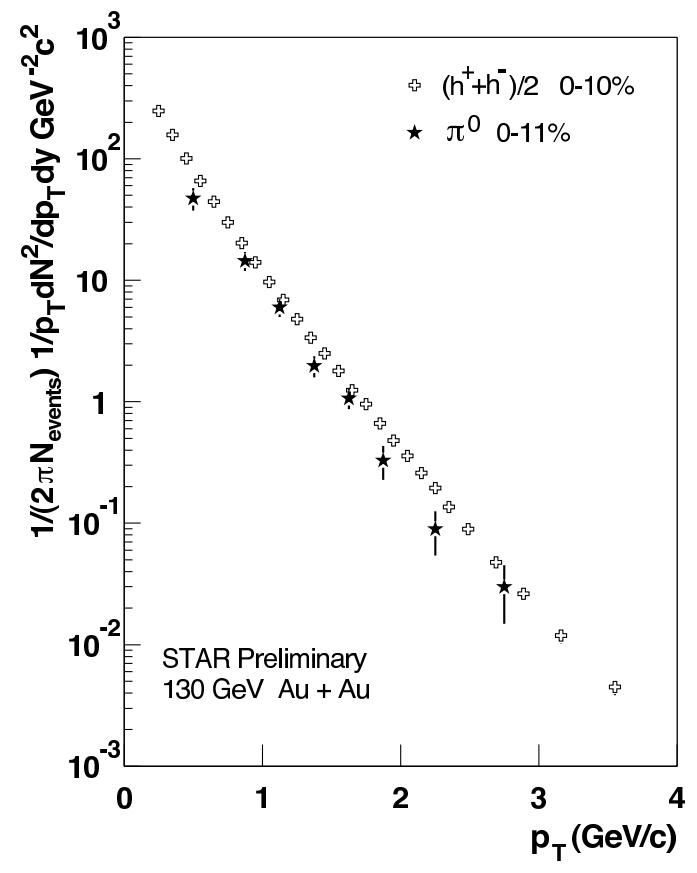

Figure 11: Spectra of $\pi^{0}$ and $\mathrm{h}^{+,-}$from central $A u+A u$ collisions at $\sqrt{s_{\mathrm{NN}}}=130$ $\mathrm{GeV}$ in STAR. Errors shown on $\pi^{0}$ data are statistical and do not include an overall normalization systematic uncertainty of $\pm 49 \%$. For $\pi^{0}$ spectra from other centralities and further details, see Ref. [0]. hadron yields provide additional information about initial conditions for particle production. Further investigations into photon spectra may help us understand the early environment of the collision system.

Particle yields seem, for the most part, to be well-described phenomenologically by statistical models. The data support thermal, chemical equilibrium fits to the yields. Such fits proffer a system with thermodynamic properties of temperature and baryo-chemical potential in the region of the phase change predicted by lattice QCD calculations.

Evidence for transverse radial flow is observed in the transverse momentum spectra of observed particles. This is consistent with the observation of strong elliptic flow by STAR [ 35]. In addition, apparently slightly suppressed $K^{* 0}$ yields hint at hadronic rescattering which is short in duration if regeneration of the $K^{* 0}$ resonance is much less than the losses caused by rescattering of in-medium decay daughters.

The STAR Experiment at RHIC continues to address a wide variety of physics topics in heavy ion and nucleon collisions. Topics explored through measurements in the soft physics regime are strengths of the experiment and will persist in providing a better understanding of the collision dynamics throughout the collision evolution. 


\section{REFERENCES}

1. J. Klay for the STAR Collaboration, these proceedings.

2. O. Barannikova and F. Wang for the STAR Collaboration, Ibid.

3. J. Castillo for the STAR Collaboration, Ibid.

4. C. Suire for the STAR Collaboration, Ibid.

5. E. Yamamoto for the STAR Collaboration, Ibid.

6. P. Fachini for the STAR Collaboration, Ibid.

7. I. Johnson for the STAR Collaboration, Ibid.

8. F. Meissner for the STAR Collaboration, Ibid.

9. C. Adler et al., Phys. Rev. Lett. 87 (2001) 112303.

10. C. Adler et al., Phys. Rev. Lett. 89 (2002) 202301.

11. L. McLerran et al., Phys. Lett. B514 (2001) 29; J. Schaffner-Bielich et al., nuclth/0202054.

12. N. Xu and $\mathrm{Z}$. Xu, these proceedings.

13. D. Kharzeev, Ibid.

14. A. Müller, Ibid.

15. T. Ullrich, Ibid.

16. P. Steinberg, Ibid.; M. Baker, Ibid.

17. C. Adler et al., Phys. Rev. Lett. 89 (2002) 092301.

18. B. Müller, private communications.

19. S. Vance, M. Gyulassy, and X.N. Wang, Phys. Lett. B443 (1998) 45.

20. J. Rafelski and B. Müller, Phys. Rev. Lett. 48 (1982) 1066; P. Koch and J. Rafelski, Nucl. Phys. A444 (1985) 678.

21. T. Matsui and H. Satz, Phys. Lett. B178 (1986) 416.

22. M. Kaneta and N. Xu, J. Phys. G27 (2001) 589.

23. J. Letessier, J. Rafelski, amd A. Tounsi, Phys. Lett. B328 (1994) 499; M. Kaneta et al.(NA44 Collaboration), J. Phys. G23 (1997) 1865.

24. P. Braun-Munzinger et al., Phys. Lett. B518 (2001) 41; W. Broniowski, A. Baran, and W. Florkowski, hep-ph/0209286; J. Rafelski and J. Letessier, these proceedings.

25. V. Koch, these proceedings.

26. Z. Fodor and S.D. Katz, nucl-th/0201071.

27. F. Antinori et al.(WA97 Collaboration), Eur. Phys. J. C14 (2000) 633; S.V. Afanasev et al.(NA49 Collaboration), Phys. Lett. B491 (2000) 59.

28. N. Xu et al.(NA44 Collaboration), Nucl. Phys. A610 (1996) 175c; I.G. Bearden et al.(NA44 Collaboration), Phys. Rev. Lett. 78 (1997) 2080;

29. A. Dumitru and D.H. Rischke, Phys. Rev. C59 (1999) 354.

30. E. Schnedermann, J. Sollfrank, and U. Heinz, Phys. Rev. C48 (1993) 2462.

31. M. van Leeuwen for the NA49 Collaboration, these proceedings.

32. G. Torrieri and J. Rafelski, Phys. Lett. B509 (2001) 239.

33. F. Retiere for the STAR Collaboration, these proceedings; L. Ray for the STAR Collaboration, Ibid.; R. Wilson for the STAR Collaboration, Ibid.

34. M.M. Aggarwal et al., Phys. Rev. Lett. 85 (2000) 3595.

35. K.H. Ackermann et al., Phys. Rev. Lett. 86 (2001) 402; C. Adler et al., Phys. Rev. Lett. 87 (2001) 182301. 


\begin{tabular}{|c|c|c|c|c|c|c|}
\hline Species & Centrality & $m_{T^{-}} m_{0}\left(\mathrm{GeV} / c^{2}\right)$ & Extrapolated $d N / d y$ & $T(\mathrm{MeV})$ & $\left\langle p_{T}\right\rangle(\mathrm{MeV} / c)$ & $\chi^{2} /$ dof \\
\hline \multirow{4}{*}{$\pi^{-}$} & $0-5 \%$ & \multirow{3}{*}{$0.10-0.62$} & $353 \pm 15 \%$ & $218 \pm 10 \%$ & $408 \pm 10 \%$ & $95 / 9$ \\
\hline & $5-10 \%$ & & $270 \pm 15 \%$ & $219 \pm 10 \%$ & $410 \pm 10 \%$ & $32 / 9$ \\
\hline & $10-20 \%$ & & $204 \pm 15 \%$ & $216 \pm 10 \%$ & $406 \pm 10 \%$ & $53 / 9$ \\
\hline & $70-80 \%$ & $0.10-0.67$ & $11.2 \pm 15 \%$ & $185 \pm 10 \%$ & $357 \pm 10 \%$ & $14 / 10$ \\
\hline \multirow{4}{*}{$K^{-}$} & $0-5 \%$ & \multirow{4}{*}{$0.04-0.40$} & $\overline{55.1 \pm 0.7 \pm 15 \%}$ & $327 \pm 5 \pm 10 \%$ & $771 \pm 10 \pm 10 \%$ & $36 / 9$ \\
\hline & $5-10 \%$ & & $44.4 \pm 0.6 \pm 15 \%$ & $326 \pm 5 \pm 10 \%$ & $769 \pm 10 \pm 10 \%$ & $44 / 9$ \\
\hline & $10-20 \%$ & & $32.1 \pm 0.3 \pm 15 \%$ & $310 \pm 3 \pm 10 \%$ & $738 \pm 6 \pm 10 \%$ & $43 / 9$ \\
\hline & $70-80 \%$ & & $1.31 \pm 0.02 \pm 15 \%$ & $202 \pm 5 \pm 10 \%$ & $531 \pm 10 \pm 10 \%$ & $16 / 9$ \\
\hline$\rho$ & $40-80 \%$ & $0.03-0.41$ & $6.59 \pm 1.08 \pm 30 \%$ & $207 \pm 54 \pm 30 \%$ & $615 \pm 110 \pm 30 \%$ & $1.63 / 5$ \\
\hline \multirow{3}{*}{$\left(K^{* 0}+\overline{K^{* 0}}\right) / 2$} & $0-20 \%$ & $0.08-2.43$ & $8.35 \pm 0.99 \pm 25 \%$ & $459 \pm 32 \pm 10 \%$ & $1141 \pm 61 \pm 10 \%$ & $11.2 / 5$ \\
\hline & $20-70 \%$ & $0.08-2.04$ & $2.68 \pm 0.18 \pm 25 \%$ & $447 \pm 20 \pm 10 \%$ & $1118 \pm 38 \pm 10 \%$ & $3.76 / 4$ \\
\hline & $70-95 \%$ & $0.08-2.04$ & $0.28 \pm 0.03 \pm 25 \%$ & $350 \pm 23 \pm 10 \%$ & $931 \pm 45 \pm 10 \%$ & $6.35 / 4$ \\
\hline \multirow{4}{*}{$\bar{p}$} & $0-5 \%$ & \multirow{4}{*}{$0.06-0.58$} & $2828.7 \pm 0.3 \pm 25 \%$ & $894 \pm 6 \pm 10 \% \ddagger$ & 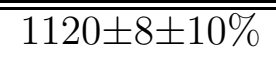 & $7 \overline{71 / 15}$ \\
\hline & $5-10 \%$ & & $22.9 \pm 0.2 \pm 25 \%$ & $861 \pm 6 \pm 10 \% \ddagger$ & $1079 \pm 8 \pm 10 \%$ & $81 / 15$ \\
\hline & $10-20 \%$ & & $16.9 \pm 0.1 \pm 20 \%$ & $830 \pm 4 \pm 10 \% \ddagger$ & $1040 \pm 5 \pm 10 \%$ & $49 / 15$ \\
\hline & $70-80 \%$ & & $0.84 \pm 0.01 \pm 15 \%$ & $550 \pm 5 \pm 10 \% \ddagger$ & $689 \pm 6 \pm 10 \%$ & $38 / 15$ \\
\hline $\mathrm{f}_{0}$ & $40-80 \%$ & $0.02-0.35$ & $1.73 \pm 0.96 \pm 50 \%$ & $200 \pm 177 \pm 50 \%$ & $652_{-460}^{+355} \pm 50 \%$ & $1.37 / 5$ \\
\hline \multirow{4}{*}{$\phi$} & $0-10 \%$ & $0.08-2.53$ & $5.9 \pm 0.5_{-0.4}^{+1.0}$ & $322 \pm 20_{-10}^{+25}$ & $909 \pm 39_{-20}^{+49}$ & $15.6 / 9$ \\
\hline & $10-30 \%$ & $0.08-2.91$ & $3.4 \pm 0.2_{-0.3}^{+0.5}$ & $337 \pm 16_{-20}^{+20}$ & $939 \pm 32_{-39}^{+39}$ & $24.4 / 10$ \\
\hline & $30-50 \%$ & $0.08-2.72$ & $1.4 \pm 0.1_{-0.1}^{+0.2}$ & $375 \pm 16_{-50}^{+10}$ & $1014 \pm 31_{-98}^{+20}$ & $20.5 / 10$ \\
\hline & $50-80 \%$ & $0.08-2.91$ & $0.40 \pm 0.02_{-0.02}^{+0.08}$ & $334 \pm 15_{-40}^{+10}$ & $953 \pm 30_{-79}^{+20}$ & $12.5 / 10$ \\
\hline$\Omega^{-}$ & $0-10 \%$ & $0.10-2.60$ & $0.31 \pm 0.04 \pm 15 \%$ & $417 \pm 23 \pm 15 \%$ & $1269 \pm 47 \pm 15 \%$ & $0.4 / 3$ \\
\hline $\bar{\Omega}^{+}$ & $0-10 \%$ & $0.10-2.60$ & $0.33 \pm 0.05 \pm 15 \%$ & $394 \pm 19 \pm 15 \%$ & $1222 \pm 39 \pm 15 \%$ & $1.2 / 3$ \\
\hline
\end{tabular}

Table 1: Preliminary results on midrapidity measurements from $\sqrt{s_{\mathrm{NN}}}=200 \mathrm{GeV} A u+A u$ collisions in STAR. The most central and most peripheral measurements available are shown. Inverse slope $T,\left\langle p_{T}\right\rangle$, and $\chi^{2} /$ dof are from $m_{T}$ exponential fits, except for Bose-Einstein on $\pi^{-}$and $p_{T}$ Gaussian on $\bar{p}$ ( $\ddagger$ where $T$ is replaced by the Gaussian width $\sigma$ ). First error quoted is statistical, second is systematic; if only one error is quoted, the error is dominated by systematics. $\chi^{2} /$ dof is large for $\pi^{-}, K^{-}$, and $\bar{p}$ due to point-to-point systematics which are much larger than the statistical errors. 